\title{
Krüppel-like factor 5 in human breast carcinoma: a potent prognostic factor induced by androgens
}

\author{
Kiyoshi Takagi, Yasuhiro Miki ${ }^{1}$, Yoshiaki Onodera ${ }^{1}$, Yasuhiro Nakamura ${ }^{1}$, \\ Takanori Ishida², Mika Watanabe ${ }^{3}$, Satoshi Inoue ${ }^{4}$, Hironobu Sasano ${ }^{1,3}$ \\ and Takashi Suzuki
}

\author{
Department of Pathology and Histotechnology, Tohoku University Graduate School of Medicine, 2-1 Seiryo-machi, Aoba-ku, Sendai, \\ Miyagi-ken 980-8575, Japan \\ Departments of ${ }^{1}$ Anatomic Pathology and ${ }^{2}$ Surgical Oncology, Tohoku University Graduate School of Medicine, Sendai 980-8575, \\ Japan \\ ${ }^{3}$ Department of Pathology, Tohoku University Hospital, Sendai 980-8574, Japan \\ ${ }^{4}$ Department of Anti-aging Medicine, Graduate School of Medicine, University of Tokyo, Tokyo 113-8655, Japan \\ (Correspondence should be addressed to K Takagi; Email: k-takagi@med.tohoku.ac.jp)
}

\begin{abstract}
Krüppel-like factor 5 (intestinal) or Krüppel-like factor 5 (KLF5) is a zinc finger-containing transcription factor and involved in important biological processes including cell proliferation and differentiation. However, clinical significance of KLF5 protein has remained largely unknown in breast cancer. Therefore, in this study, we immunolocalized KLF5 in 113 human breast carcinoma cases. KLF5 immunoreactivity was frequently detected in the nuclei of breast carcinoma cells, and median value of the ratio of KLF5-positive carcinoma cells was $30 \%$ and was positively associated with the status of androgen receptor. KLF5 immunoreactivity was also significantly associated with increased risk of recurrence and worse clinical outcome in breast cancer patients by univariate analyses, and subsequent multivariate analyses demonstrated that KLF5 immunoreactivity was an independent prognostic factor for both disease-free and breast cancer-specific survival of the patients. We then examined possible regulation of KLF5 by androgen using MCF-7 breast carcinoma cells. KLF5 mRNA was induced by biologically active androgen $5 \alpha$-dihydrotestosterone in a dose- and time-dependent manner in MCF-7 cells. In addition, results of transfection experiments demonstrated that proliferation activity of MCF-7 cells was significantly associated with the KLF5 expression level. These findings suggest that $K L F 5$ is an androgen-responsive gene in human breast carcinomas and play important roles in the progression of breast carcinomas. KLF5 immunoreactivity is therefore considered a potent prognostic factor in human breast cancers.
\end{abstract}

Endocrine-Related Cancer (2012) 19 741-750

\section{Introduction}

Breast cancer is one of the most common malignancies in women worldwide. Human breast tissue is a target of sex steroids, and both estrogens and androgens play important roles in the development and/or progression of hormone-responsive breast carcinomas.

Krüppel-like factor 5 (intestinal) or Krüppel-like factor $5(K L F 5)$ is a zinc finger-containing transcription factor and ubiquitously expressed in various human tissues such as breast, stomach, and colon (Conkright et al. 1999, Shi et al. 1999, Chen et al. 2002, 2003). KLF5 is also known to be involved in important biological processes such as cell proliferation and differentiation (Bieker 2001, Black et al. 2001), and its expression is induced by growth stimuli, consistent with its potent pro-proliferating roles (Bieker 2001, Sun et al. 2001). Recently, KLF5 has been proposed to be involved not only in normal human tissues but also in their malignant tumors (Tong et al. 2006, Kwak et al. 2007, Chen et al. 2011, Nakajima et al. 2011, Soon et al. 2011). However, its biological functions and/or clinical significance in human carcinomas have remained largely unknown, which is partly due to the fact that results of previous reports varied among different types of carcinomas 
(reviewed in Dong \& Chen (2009)). For instances, Kwak et al. (2007) reported that KLF5 immunoreactivity was detected in only $47 \%$ of gastric caner patients, while it was detected in a majority of normal stomach, and demonstrated that KLF5 immunoreactivity was a favorable prognostic factor in gastric cancer. However, Soon et al. (2011) recently demonstrated KLF5 as a worse prognostic factor in gastric cancer using immunohistochemistry. KLF5 was also reported to inhibit cell proliferation of esophageal carcinoma cells (Yang et al. 2005), but its expression was also reported to be associated with activating $K R A S$ mutations and to promote both tumorigenesis and cell proliferation in the colorectal carcinomas (Nandan et al. 2008).

In breast cancer, KLF5 was reported to promote breast carcinoma cell proliferation by inducing fibroblast binding protein 1 (Liu et al. 2009, Zheng et al. 2009). In addition, Liu et al. (2011) reported that KLF5 was involved in progesterone-dependent breast carcinoma cell proliferation. Tong et al. (2006) also demonstrated KLF5 mRNA as a prognostic factor in human breast cancers using 90 breast cancer specimens. However, it is also true that KLF5 protein is rapidly degraded by ubiquitin-proteasome system (Chen et al. 2005, Liu et al. 2010, Zhao et al. 2010), and KLF5 mRNA level may not always reflect its biological functions. Overexpression of KLF5 protein in breast carcinoma tissues has been reported by Chen et al. (2011), but it remains unclear whether KLF5 protein can be a prognostic factor or not in the patients with breast cancer as reported in other human malignancies. Therefore, in this study, we first immunolocalized KLF5 in breast cancer tissues and correlated the findings with various clinicopathological factors to assess clinicopathological significance of KLF5 in breast cancer patients.

\section{Materials and methods}

\section{Patients and tissues}

One hundred and thirteen specimens of invasive ductal carcinoma of human breast were obtained from women who underwent surgical treatment from 1990 to 1999 in the Department of Surgery, Tohoku University Hospital, Sendai, Japan. All the patients did not receive chemotherapy, irradiation, or hormonal therapy before the surgery. The clinical outcome of the patients was evaluated by both disease-free and breast cancerspecific survival. The mean age was 56 years (range 31-81 years), and the mean follow-up time was 103 months (range 1-175 months). All the specimens had been fixed in $10 \%$ formalin and embedded in paraffin wax. Research protocols for this study were approved by the Ethics Committee at Tohoku University School of Medicine (approval number 2008-288).

\section{Immunohistochemistry}

Characteristics of primary antibody for KLF5 were reported previously (Kwak et al. 2007). Mouse monoclonal antibodies for estrogen receptor (ER; ER1D5), progesterone receptor (PR; MAB429), androgen receptor (AR; AR441), and Ki-67 (MIB1) were purchased from Immunotech (Marseille, France), Chemicon (Temecula, CA, USA), and DAKO (Carpinteria, CA, USA) respectively. Rabbit polyclonal antibody for HER2 (A0485) was purchased from DAKO. A Histofine Kit (Nichirei, Tokyo, Japan), which employs streptavidin-biotin amplification method, was used. The antigen-antibody complex was visualized with $3,3^{\prime}$-diaminobenzidine and counterstained with hematoxylin. Human tissue of the stomach was used as a positive control for KLF5 antibody (Kwak et al. 2007), and PBS was used instead of the primary antibody as a negative control of KLF5 immunostaining in this study.

\section{Scoring of immunoreactivity and statistical analyses}

KLF5, ER, PR, AR, and Ki-67 immunoreactivity was detected in the nucleus of breast carcinoma cells, and the immunoreactivity was evaluated in more than 1000 carcinoma cells for each case, and subsequently, the percentage of immunoreactivity, i.e. labeling index (LI), was determined. KLF5 immunoreactivity was subsequently classified into two groups (i.e. high and low groups) according to the median value of KLF5 LI (Ferreira et al. 2001). Cases with ER LI of more than $1 \%$ were considered ER-positive breast carcinoma, according to a previous report (Hammond et al. 2010). HER2 immunoreactivity was evaluated according to a grading system proposed in HercepTest (DAKO), and strongly circumscribed membrane staining of HER2 in more than $10 \%$ carcinoma cells was considered positive in this study.

An association between KLF5 immunoreactivity and clinicopathological factors was evaluated using one-way ANOVA and Bonferroni test or a crosstable using $\chi^{2}$ test. Breast cancer-specific and disease-free survival curves were generated according to Kaplan-Meier method; statistical significance was calculated using log-rank test. Univariate and multivariate analyses were evaluated by proportional hazard model (COX). $P$ values $<0.05$ were considered significant in this study. 


\section{Cell line and chemicals}

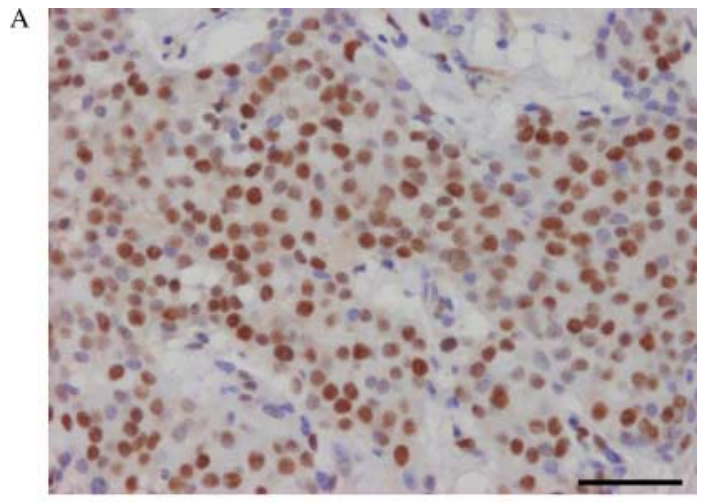

B

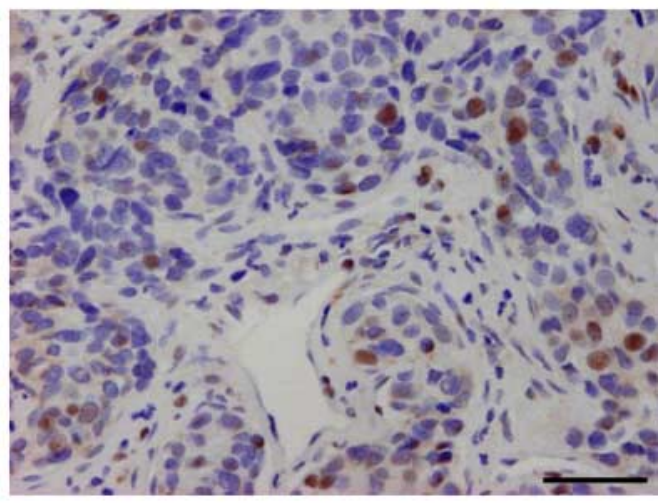

C

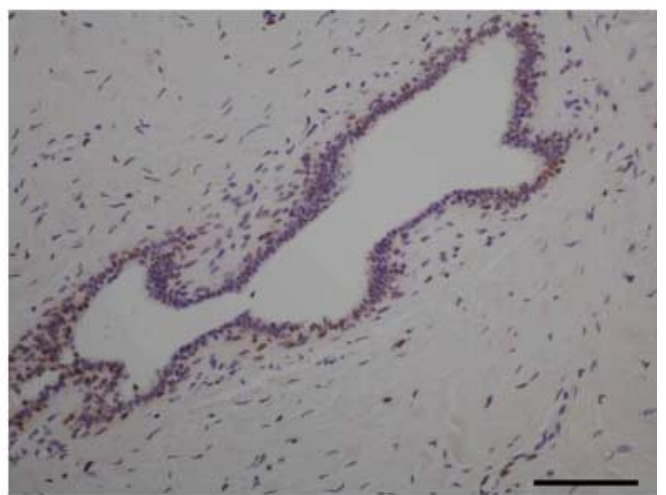

D

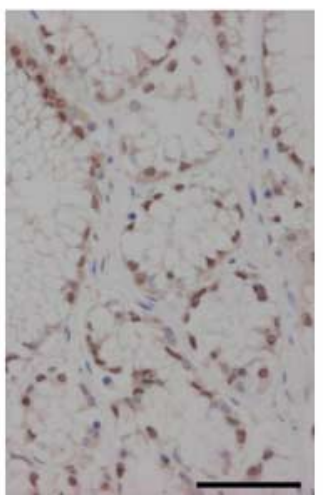

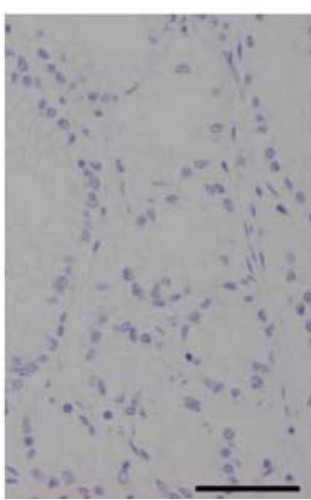

Human breast cancer cell line MCF-7 was provided from Cell Resource Center for Biomedical Research, Tohoku University (Sendai, Japan), and cultured in RPMI 1640 (Sigma-Aldrich) with 10\% fetal bovine serum (FBS; FJRH Bioscience, Lenexa, KS, USA). MCF-7 cells were cultured in phenol-red-free RPMI 1640 with $10 \%$ dextran-coated charcoal-stripped FBS for 3 days before the treatment of sex steroids.

\section{Real-time PCR}

Total RNA was extracted using TRIzol reagent (Invitrogen), and cDNA was synthesized using a QuantiTect reverse transcription kit (Qiagen). Realtime PCR was performed using the LightCycler System and FastStart DNA Master SYBR Green I (Roche Diagnostics). The primer sequence of KLF5 and the ribosomal protein L13A (RPL13A) was as follows. KLF5: forward 5'-ATAGAAGGAGTAACCCCGAT- $3^{\prime}$ and reverse $5^{\prime}$-CACTTGTATGGCTTTTCACC- ${ }^{\prime}$; and RPL13A: forward 5'-CCTGGAGGA-GAAGAGGAAAGAGA-3' and reverse 5'-TTGAGGACCTCTGTGTATTTGTCAA-3' (Takagi et al. 2010). KLF5 mRNA level was calculated as the ratio of RPL13A mRNA level.

\section{Luciferase assay}

In this study, two androgen-responsive reporter plasmids were used (i.e. pKLF5-ARE-Luc and pPSAE-Luc plasmids). At first, pKLF5-ARE-Luc plasmids were constructed to examine possible recruitment of $A R$ into putative androgen-responsive element(s) (ARE) in first intron of KLF5 gene identified by Frigo et al. (2009). Briefly, $158 \mathrm{bp}$ DNA region containing ARE within was amplified by PCR method using adapter primers (forward 5'-TAGGTACCTCAGTGGATACAAGGTTTTGC-3' and reverse $5^{\prime}$-TATAAGCTTCGATGCAAACCTTTAAAATATTGC- $3^{\prime}$ ). DNA fragment was digested with HindIII and KpnI and cloned into pGL3 basic luciferase reporter vectors (Promega). Secondly, we used pPSAE-Luc, androgen-responsive reporters,

Figure 1 Immunohistochemistry for KLF5 in the breast carcinoma. (A and B) Representative picture of KLF5-high (A) and KLF5-low (B) cases. KLF5 immunoreactivity was detected in the nucleus of carcinoma cells. AR LIs in each case was $56 \%$ (A) and $2 \%$ (B) respectively. (C) KLF5 immunoreactivity was focally detected in the nucleus of epithelium in the morphologically normal mammary glands. (D) Positive control (left) and negative control (right) section of KLF5 immunohistochemistry (fundic glands of the stomach). Bar $=100 \mu \mathrm{m}$ respectively. Full colour version of this figure available via http://dx.doi.org/ 10.1530/ERC-12-0017. 
which contained ARE of KLK3 (Takagi et al. 2010) in order to examine transcriptional activity of AR.

One microgram of pKLF5-ARE-Luc or pPSAE-Luc and $200 \mathrm{ng}$ pRL-TK-control plasmids (Promega) were used to measure the transcriptional activity of endogenous AR. Transient transfections were carried out using TransIT-LT Transfection Reagents (TaKaRa, Tokyo, Japan) in MCF-7 cells, and the luciferase activity was measured using a Dual-Luciferase Reporter Assay System (Promega) and luminescencerPSN (AB-2200; Atto Co., Tokyo, Japan) after incubation with $10 \mathrm{nM} 5 \alpha$-dihydrotestosterone (DHT) for $24 \mathrm{~h}$. The transfection efficiency was normalized against Renilla luciferase activity using pRL-KT control plasmids, and the luciferase activity for each sample was evaluated as the ratio (\%) compared with that of controls.

\section{Small interfering RNA transfection}

Small interfering RNA (siRNA) oligonucleotides for KLF5 (siKLF5) were obtained from Genolution Pharmaceuticals, Inc. (Seoul, South Korea). The target sequences of siKLF5 were as follows: sense $5^{\prime}$-GUCGUAGACCAGUUCUUCAUU- $3^{\prime}$ and antisense $5^{\prime}$-UGAAGAACUGGUCUACGACUU- $3^{\prime}$. In addition, medium GC duplex \#2 (Invitrogen) was used as a negative control (siCTRL). The siRNA was transfected using HiperFect transfection reagent (Qiagen).

\section{Cell proliferation assay}

MCF-7 cells were transfected with siKLF5 or siCTRL in a 96-well culture plate. Three days after transfection, the cell proliferation was measured using Cell Counting Kit-8 (Dojindo, Kumamoto, Japan).

\section{Results}

\section{Immunolocalization of KLF5 in human breast carcinomas}

KLF5 immunoreactivity was detected in the nuclei of carcinoma cells (Fig. 1A and B), and the median LI of KLF5 was 30\% (range 2-99\%). KLF5 immunoreactivity was focally detected in the epithelial cells of morphologically normal mammary glands, but its LI (ranged from 0 to $15 \%$ ) was much lower than that in carcinoma cells (Fig. 1C). In the positive control, KLF5 immunoreactivity was detected in fundic glands of the normal stomach (left panel of Fig. 1D) as reported previously (Kwak et al. 2007), whereas no significant immunoreactivity was detected in the same area of negative control section (right panel of Fig. 1D).

KLF5 immunoreactivity was then classified into two groups according to median KLF5 LI (30\%), and cases with KLF5 LI of more than $30 \%$ were considered KLF5-high breast carcinomas in this study. Associations between KLF5 immunoreactivity and various clinicopathological parameters in 113 breast carcinomas were summarized in Table 1. Significant correlation was detected between KLF5 immunoreactivity and AR LI $(P=0.039)$, while no significant associations were detected between KLF5 immunoreactivity and other parameters examined. Significant correlation between KLF5 immunoreactivity and AR LI was also detected when KLF5 LI was used as a continuous variable $(P=0.038$ and $r=0.20$, data not shown).

Table 1 Association between KLF5 immunoreactivity and clinicopathological parameters in 113 breast carcinomas

\begin{tabular}{|c|c|c|c|}
\hline & \multicolumn{2}{|c|}{ KLF5 immunoreactivity } & \multirow[b]{2}{*}{$P$ value } \\
\hline & $\begin{array}{l}\text { High } \\
(n=57)\end{array}$ & $\begin{array}{l}\text { Low } \\
(n=56)\end{array}$ & \\
\hline Age (years) $^{\mathrm{a}}$ & $55.5 \pm 3.1$ & $57.2 \pm 3.1$ & 0.43 \\
\hline \multicolumn{4}{|l|}{ Menopausal status } \\
\hline Premenopausal & $21(19 \%)$ & $17(15 \%)$ & \\
\hline Postmenopausal & $36(32 \%)$ & $39(35 \%)$ & 0.28 \\
\hline \multicolumn{4}{|l|}{ Stage } \\
\hline $\mathrm{I}+\mathrm{II}$ & 45 & 42 & \\
\hline III+IV & 12 & 14 & 0.78 \\
\hline \multicolumn{4}{|c|}{ Pathological tumor factor (pT) } \\
\hline pT1 & $19(17 \%)$ & $25(22 \%)$ & \\
\hline pT2-4 & $38(34 \%)$ & $31(27 \%)$ & 0.30 \\
\hline \multicolumn{4}{|l|}{ Lymph node status } \\
\hline Positive & $30(27 \%)$ & $21(19 \%)$ & \\
\hline Negative & 27 (24\%) & $35(31 \%)$ & 0.15 \\
\hline \multicolumn{4}{|l|}{ Distant metastasis } \\
\hline Positive & $6(5 \%)$ & $2(2 \%)$ & \\
\hline Negative & $51(45 \%)$ & $54(48 \%)$ & 0.28 \\
\hline \multicolumn{4}{|l|}{ Histological grade } \\
\hline 1,2 (well or moderate) & 40 & 39 & \\
\hline 3 (poor) & 17 & 17 & $>0.99$ \\
\hline \multicolumn{4}{|l|}{ ER status } \\
\hline Positive & 49 (43\%) & $43(38 \%)$ & \\
\hline Negative & $8(7 \%)$ & $13(12 \%)$ & 0.31 \\
\hline ER LI (\%) ${ }^{\mathrm{a}}$ & $48.8 \pm 9.5$ & $47.4 \pm 10.0$ & 0.84 \\
\hline PR LI (\%) ${ }^{a}$ & $30.4 \pm 8.4$ & $28.2 \pm 8.1$ & 0.71 \\
\hline AR LI (\%) ${ }^{a}$ & $34.2 \pm 7.5$ & $24.3 \pm 5.8$ & 0.039 \\
\hline \multicolumn{4}{|l|}{ HER2 status } \\
\hline Positive & $13(12 \%)$ & $8(7 \%)$ & \\
\hline Negative & 44 (39\%) & $48(42 \%)$ & 0.36 \\
\hline Ki-67 LI (\%) & $19.7 \pm 3.9$ & $20.2 \pm 4.7$ & 0.86 \\
\hline
\end{tabular}

$P$ value $<0.05$ were considered significant, and described as boldface.

${ }^{a}$ Data was presented as mean $\pm 95 \%$ confidence interval $(95 \% \mathrm{Cl})$ or the number of cases with percentage. 


\section{Correlation between KLF5 immunoreactivity and clinical outcome of breast cancer patients}

In order to examine the association between KLF5 immunoreactivity and patient prognosis, we excluded stage IV cases and used stage I-III breast carcinoma patients $(n=105)$ in the following analyses. As demonstrated in Fig. 2A, KLF5 immunoreactivity was significantly associated with increased risk of recurrence $(P=0.033$ by log-rank test), and multivariate analysis revealed that lymph node metastasis $(P=0.0049)$, pathological tumor factor $(\mathrm{pT})(P=0.0498)$, and KLF5 immunoreactivity $(P=0.0095)$ were independent prognostic factors for disease-free survival with relative risks over 1.0 (Table 2).

Association between KLF5 immunoreactivity and breast cancer-specific survival was summarized in Fig. 2B. Significant association was detected between KLF5 immunoreactivity and adverse clinical outcome in 105 breast carcinoma patients examined $(P=0.014$ by log-rank test). Multivariate analysis demonstrated that only KLF5 immunoreactivity $(P=0.031)$ was an independent prognostic factor with relative risk over 1.0 (Table 3). Similar tendency was also detected when KLF5 immunoreactivity was further divided into four groups $(P=0.032$ for disease-free survival (Fig. 2C) and $P$ value was not calculated for breast cancer-specific survival because no patients died in the group with KLF5 LI of $<10 \%$ (Fig. 2D)). When KLF5 LI was used as a continuous variable, it was also indicated as an independent prognostic factor for both disease-free and breast cancer-specific survival $(P=0.0045$ and $P=0.0050$ respectively) (data not shown).

\section{KLF5 as an androgen-responsive gene in breast carcinomas}

We detected a significant correlation between KLF5 and AR in our immunohistochemical analysis as shown in Table 1, which suggested a possible regulation of KLF5 by androgens in human breast carcinoma cells.

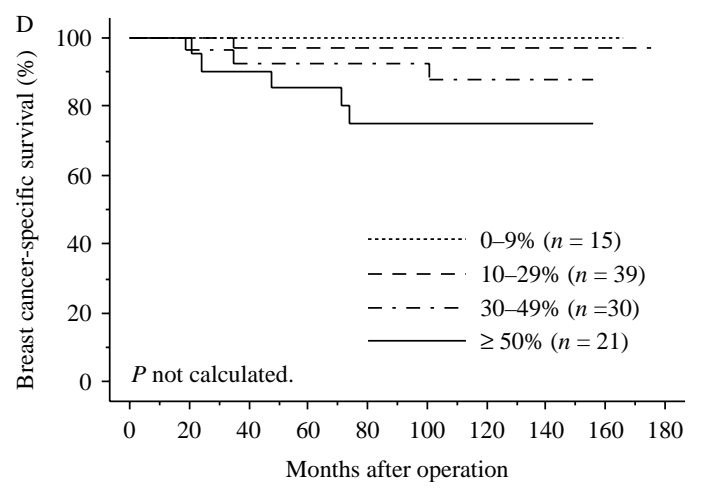

Figure 2 Disease-free and breast cancer-specific survival of 105 breast carcinoma patients according to KLF5 immunoreactivity. ( $A$ and $B)$ KLF5 immunoreactivity was significantly associated with an increased risk of recurrence $(P=0.033)(\mathrm{A})$ and worse prognosis $(P=0.014)(B)$. Solid line, high-KLF5 immunoreactivity $(n=51)$; and dashed line, low-KLF5 immunoreactivity $(n=54)$. (C and D) Disease-free (C) and breast cancer-specific (D) survival curves when KLF5 immunoreactivity was further divided into four groups (i.e. 0-9\% (dotted line), 10-29\% (dashed line), 30-50\% (dot-dashed line), and $\geq 50 \%$ (solid line) of KLF5 LI). $P$ values were evaluated by log-rank test. In D, $P$ value was not calculated because no patients died in the group with KLF5 LI of $<10 \%$. 
Table 2 Univariate and multivariate analyses of disease-free survival in stages I-III breast cancer patients examined $(n=105)$

\begin{tabular}{|c|c|c|c|}
\hline \multirow[b]{2}{*}{ Variable } & \multirow{2}{*}{$\begin{array}{c}\text { Univariate } \\
\qquad\end{array}$} & \multicolumn{2}{|c|}{ Multivariate } \\
\hline & & $\boldsymbol{P}$ & $\begin{array}{c}\text { Relative risk } \\
(95 \% \mathrm{Cl})\end{array}$ \\
\hline $\begin{array}{l}\text { Lymph node status } \\
\text { (positive/negative) }\end{array}$ & 0.0059 & 0.0049 & $\begin{array}{c}4.51 \\
(1.58-12.9)\end{array}$ \\
\hline $\begin{array}{l}\text { KLF5 immunoreactivity } \\
\text { (high/low) }\end{array}$ & 0.0075 & 0.0095 & $\begin{array}{c}4.42 \\
(1.44-13.4)\end{array}$ \\
\hline $\begin{array}{l}\text { HER2 status } \\
\text { (positive/negative) }\end{array}$ & 0.037 & 0.056 & \\
\hline pT $(2,3 / 1)$ & 0.013 & 0.0498 & $\begin{array}{c}3.51 \\
(1.00-12.3)\end{array}$ \\
\hline Ki-67 LI (82-0\%) & 0.55 & & \\
\hline $\begin{array}{l}\text { ER status } \\
\quad \text { (negative/positive) }\end{array}$ & 0.64 & & \\
\hline $\begin{array}{l}\text { Histological grade (3/1, } \\
\text { 2) }\end{array}$ & 0.95 & & \\
\hline
\end{tabular}

Data considered significant $(P<0.05)$ in the univariate analyses were described as boldface, and these parameters were examined in the multivariate analyses.

However, such findings have not been reported yet to the best of our knowledge. Therefore, we performed following in vitro experiments using MCF-7 cells, which expressed both ER and AR (Horwitz et al. 1975).

As demonstrated in Fig. 3A, KLF5 mRNA was significantly induced by bioactive androgen DHT in a dose-dependent manner, and this increment became significant from $10 \mathrm{pM}$ (1.8-fold, $P<0.05)$. However, DHT-mediated induction of KLF5 mRNA was significantly suppressed to the basal level by addition of AR antagonist bicalutamide ( 0.35 -fold compared with DHT $(10 \mathrm{nM})$ alone, $P<0.0001)$. Bicalutamide alone did not significantly ( 0.96 -fold, $P=0.71)$ change $K L F 5$ mRNA levels in MCF-7 cells. DHT $(10 \mathrm{nM})$ also induced KLF5 mRNA in MCF-7 cells in a timedependent fashion (Fig. 3B), and the induction became significant after $8 \mathrm{~h}$ from DHT treatment (1.7-fold, $P<0.05)$. Similar results were obtained when other breast carcinoma cell line T-47D, which expressed both ER and AR (Migliaccio et al. 2000), was used (data not shown). On the other hand, estradiol did not significantly influence KLF5 mRNA expression in MCF-7 cells (1.2-fold, $P=0.51$; data not shown).

In order to examine whether $K L F 5$ was a primary responsive gene of androgen or not, MCF-7 cells were treated together with DHT and protein synthesis inhibitor cycloheximide. As demonstrated in Fig. 3C, cycloheximide did not completely suppress DHTmediated induction of KLF5 (2.5-fold, $P<0.001$, compared with control group).
We next performed luciferase assay to examine possible recruitment of AR into ARE of KLF5 gene previously identified by Frigo et al. (2009) using pKLF5-ARE-Luc plasmids. The luciferase activity was not significantly increased by DHT (0.99-fold, $P=0.93)$ in MCF-7 cells, although it was 1.2-fold $(P=0.04)$ increased in T-47D cells (Fig. 3D).

\section{Effects of KLF5 on breast cancer cell proliferation and $A R$ function}

The results of our study suggested that KLF5 was associated with worse prognosis of breast carcinoma patients (Fig. 2). In order to further examine the effects of KLF5 on breast cancer cell proliferation, we transfected specific siRNA for KLF5 (siKLF5) in MCF-7 cells. KLF5 mRNA level was markedly (0.54-fold, $P<0.001)$ suppressed in the cells transfected with siKLF5 compared with those transfected with nonspecific control siRNA (siCTRL) at the 3 days after the transfection (Fig. 4A). As shown in Fig. 4B, cell proliferation activity was significantly suppressed in the cells transfected with siKLF5 compared with those transected with siCTRL $(0.67$-fold, $P<0.05)$.

We also found out a positive association between KLF5 and AR immunoreactivity in breast carcinoma tissues (Table 1) and upregulation of KLF5 mRNA expression by androgens. Therefore, it may be possible to speculate the modulation of AR function by KLF5. To assess this hypothesis, we performed luciferase assay using ARE reporter plasmids pPSAE-luc. However, DHT-mediated luciferase activity of MCF-7 cells was not significantly $(P=0.86)$ changed between siCTRL and siKLF5 groups (Fig. 4C).

Table 3 Univariate and multivariate analyses of breast cancerspecific survival in stage I-III breast cancer patients examined $(n=105)$

\begin{tabular}{|c|c|c|c|}
\hline \multirow[b]{2}{*}{ Variable } & \multirow{2}{*}{$\begin{array}{c}\text { Univariate } \\
\qquad P\end{array}$} & \multicolumn{2}{|c|}{ Multivariate } \\
\hline & & $P$ & $\begin{array}{c}\text { Relative risk } \\
(95 \% \mathrm{Cl})\end{array}$ \\
\hline $\begin{array}{l}\text { Lymph node status } \\
\text { (positive/negative) }\end{array}$ & 0.15 & & \\
\hline $\begin{array}{l}\text { KLF5 immunoreactivity } \\
\text { (high/low) }\end{array}$ & 0.042 & 0.031 & $\begin{array}{l}11.5 \\
\quad(1.24-107)\end{array}$ \\
\hline $\begin{array}{l}\text { HER2 status } \\
\text { (positive/negative) }\end{array}$ & 0.0042 & 0.32 & \\
\hline pT $(2,3 / 1)$ & 0.074 & & \\
\hline Ki-67 LI (82-0\%) & 0.0097 & 0.60 & \\
\hline $\begin{array}{l}\text { ER status } \\
\quad \text { (negative/positive) }\end{array}$ & 0.019 & 0.41 & \\
\hline Histological grade $(3 / 1,2)$ & 0.0046 & 0.14 & \\
\hline
\end{tabular}

Data considered significant $(P<0.05)$ in the univariate analyses were described as boldface, and these were analyzed in the multivariate analyses. 


\section{Discussion}

This is the first study that demonstrated the association between KLF5 immunoreactivity and clinical outcome of breast carcinomas to the best of our knowledge. KLF5 immunoreactivity was detected in $50 \%$ of breast
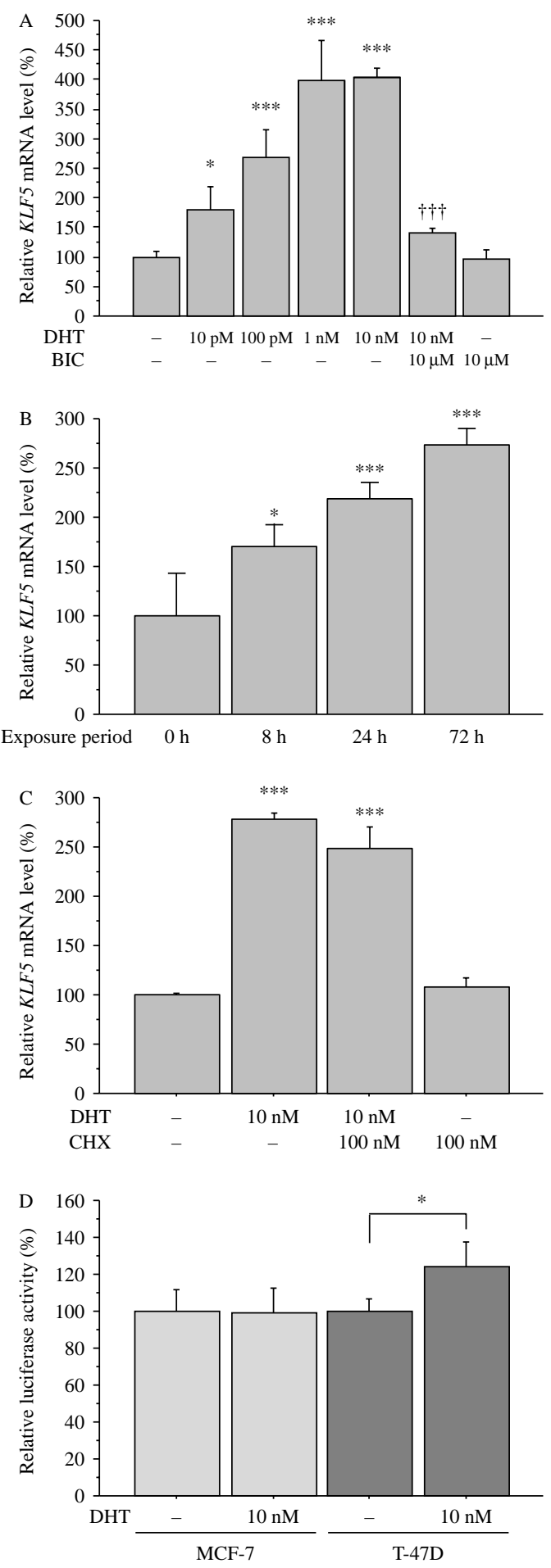

cancer cases and was increased compared with morphologically normal mammary glands. KLF5 immunoreactivity was also significantly associated with increased risk of recurrence and poor prognosis in breast cancer patients, and subsequent multivariate analyses revealed that KLF5 immunoreactivity was an independent prognostic factor for both recurrence and breast cancer-specific survival of the patients. These findings are in good agreement with the previous report by Tong et al. (2006), which demonstrated significant association between KLF5 mRNA level and poor prognosis of breast cancer patients. Results of subsequent in vitro experiments demonstrated that KLF5 significantly promoted breast cancer cell proliferation. These findings indicated possible roles of KLF5 in the progress of breast carcinomas. In prostate carcinomas, however, KLF5 is considered a better prognostic marker (Nakajima et al. 2011) and expression of KLF5 was also reported to be suppressed in prostate cancer (Chen et al. 2003). These differences among different types of human malignancies may be partly explained by diversity of the expression patterns of KLF5 responsive genes because KLF5 is known to regulate several different genes associated with cell proliferation (reviewed in Dong \& Chen (2009)). Bateman et al. (2004) reported that KLF5 mediated cyclin D1 expression in untransformed intestinal epithelial cells, while cyclin D1 was also considered a poor prognostic factor in breast carcinomas (Kenny et al. 1999). KLF5 was also reported to be essential for the expression of c-myc (Guo et al. 2009), which was considered poor prognostic factor in breast cancer patients (Berns et al. 1992). Therefore, KLF5 may contribute to the progression of breast carcinomas by regulating genes associated with poor prognosis of breast cancer patients but further examinations of KLF5 responsive genes are required to clarify the molecular mechanisms of KLF5 in the breast carcinoma or its tissue-specific functions.

Figure 3 Induction of KLF5 expression by DHT in MCF-7 breast carcinoma cells. (A, B and C) Effects of DHT on KLF5 mRNA expression were examined by real-time PCR analysis. MCF-7 cells were treated with indicated concentrations of DHT with or without an AR blocker bicalutamide (BIC) (A) or cycloheximide $(\mathrm{CHX})(\mathrm{C})$ for $72 \mathrm{~h}$, treated with DHT $(10 \mathrm{nM})$ for indicated period (B). KLF5 mRNA level was evaluated as a ratio of $R P L 13 A$ mRNA level, and subsequently relative KLF5 mRNA level was summarized a ratio (\%) compared with the basal level (nontreatment). (D) Recruitment of AR into ARE was examined by luciferase assay. MCF-7 and T-47D cells were treated with DHT (10 nM) for $24 \mathrm{~h}$. Relative luciferase activity was evaluated as a ratio (\%) of basal level (nontreatment). Data were presented as the mean \pm S.D. $(n=3)$ respectively. ${ }^{*} P<0.05$, ${ }^{\star \star \star} P<0.001$ vs nontreatment (left column), and ${ }^{{ }^{\dagger \dagger} P} P<0.001$ vs DHT (10 nM)-treated group. The statistical analyses were performed using one-way ANOVA and Bonferroni tests. 

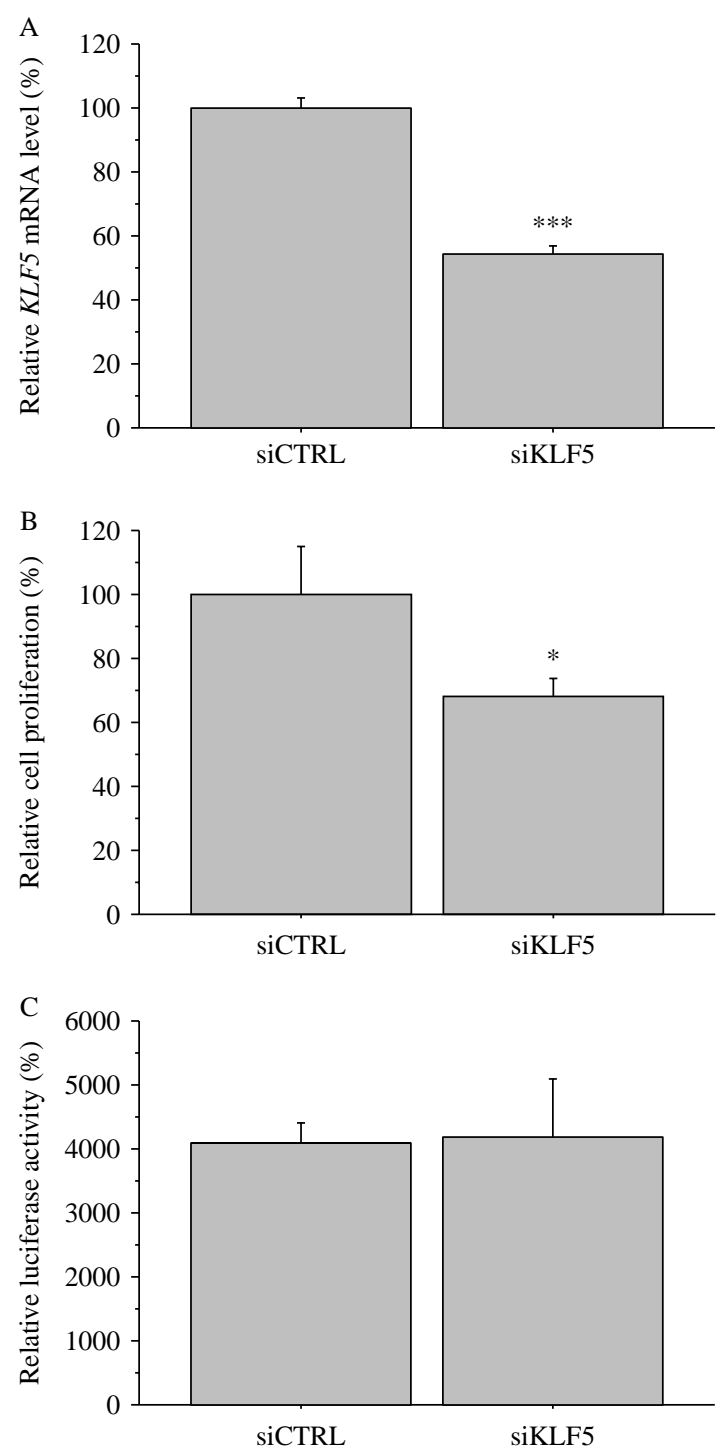

Figure 4 Effects of KLF5 on breast carcinoma cell proliferation and AR function. (A) Knockdown of KLF5 mRNA level in MCF-7 cells transfected with specific siRNA for KLF5 (siKLF5) compared with those transfected with nonspecific control siRNA (siCTRL) was examined by real-time PCR analysis. KLF5 mRNA level was evaluated as a ratio of RPL13A mRNA level, and subsequently, relative KLF5 mRNA level was summarized a ratio (\%) compared with siCTRL group. (B) Relative cell proliferation was evaluated as a ratio (\%) compared with siCTRL group. (C) DHT-dependent transactivation was examined by luciferase assay. Relative luciferase activity was evaluated as a ratio (\%) compared with SiCTRL group. Data were presented as the mean \pm s.D. $(n=3)$ respectively. ${ }^{\star} P<0.05$ and ${ }^{* \star \star} P<0.001$ vs nontreatment (left column). The statistical analyses were performed using one-way ANOVA and Bonferroni tests.

In our present study, the status of KLF5 immunoreactivity was significantly correlated with AR LI. Therefore, we examined possible regulation of KLF5 by androgen using in vitro studies. Real-time PCR analyses revealed that $K L F 5$ mRNA was significantly induced by DHT in both dose- and time-dependent manners. Moreover, cycloheximide did not affect DHT-mediated induction of KLF5 mRNA level, suggesting that $K L F 5$ was a primary responsive gene of androgen. In consistent with our present results Frigo et al. (2009), previously reported a direct induction of KLF5 mRNA expression by R1881, synthetic androgen, in a prostate cancer cell line (LNCaP). They also identified putative ARE in first intron of KLF5 gene. However, subsequent luciferase assay revealed that this ARE was not necessarily activated by endogenous AR in the breast carcinoma cells in our study. As five potential AREs were found in $-4 \mathrm{kbp}$ upstream region of KLF5 gene (http://www. cbil.upenn.edu/cgi-bin/tess/tess), KLF5 expression may be regulated by androgen through several AREs in the promoter region in the breast carcinoma cells. In addition, it is also true that Liu et al. (2011) reported an induction of KLF5 by progesterone in breast cancer cell line (T-47D). In our present study, $25 \%$ of high KLF5 immunoreactivity breast cancers (14 out of 57 cases) were associated with negative AR status (i.e. AR LI < 10\%) (data not shown). Therefore, KLF5 expression may be regulated by several factors other than androgen and may not necessarily reflect the sequels of androgen actions in the breast carcinomas.

Pro-proliferating roles of androgens have been recently reported by several groups, although androgens are generally considered to exert antiproliferative effects in breast carcinomas (reviewed in Suzuki et al. (2010)). Especially, functional cross talk between AR and HER2 signaling has been proposed in ER-negative/HER2-positive breast carcinomas (Naderi \& Hughes-Davies 2008, Ni et al. 2011). Naderi \& Hughes-Davies (2008) demonstrated that growth stimulatory effect of heregulin, a ligand of HER1 and HER3, was inhibited by AR inhibitor flutamide in MDA-MB-453 cells. On the other hand, Gregory et al. (2005) demonstrated that heregulin-induced activation of HER2 and HER3 increased AR transactivation and Beckers et al. (2005) previously reported that overexpression of HER2 induced KLF5 expression using microarray analysis. Taken together with these previous findings and our present results, pro-proliferating effects of androgens may be, at least in a part, mediated by induction of KLF5 through AR in the breast carcinoma. Multicenter phase II trial of bicalutamide for ER-negative/AR-positive metastatic breast cancer are ongoing (NCT00468715), and our present study may serve as a starting point for clarification of molecular mechanisms and/or predictive marker of AR-based therapy in the breast carcinoma patients. 
In summary, KLF5 immunoreactivity was frequently detected in breast cancer tissues, and its immunoreactivity was positively associated with AR LI. KLF5 immunoreactivity was significantly associated with increased risk of recurrence and worse prognosis of breast carcinoma patients. Subsequent in vitro studies revealed that $K L F 5$ mRNA was induced by DHT and KLF5 expression level was associated with the proliferation activity in MCF-7 cells. These results suggested that KLF5 was an androgenresponsive gene and plays important roles in the progression of breast carcinomas.

\section{Declaration of interest}

The authors declare that there is no conflict of interest that could be perceived as prejudicing the impartiality of the research reported.

\section{Funding}

This research did not receive any specific grant from any funding agency in the public, commercial, or not-for-profit sector.

\section{Acknowledgements}

We appreciate skillful technical assistance of Mr Katsuhiko Ono (Department of Anatomic pathology, Tohoku University Graduate School of Medicine).

\section{References}

Bateman NW, Tan D, Pestell RG, Black JD \& Black AR 2004 Intestinal tumor progression is associated with altered function of KLF5. Journal of Biological Chemistry 279 12093-12101. (doi:10.1074/jbc. M311532200)

Beckers J, Herrmann F, Rieger S, Drobyshev AL, Horsch M, Hrabé de Angelis M \& Seliger B 2005 Identification and validation of novel ERBB2 (HER2, NEU) targets including genes involved in angiogenesis. International Journal of Cancer 114 590-597. (doi:10.1002/ijc.20798)

Berns EM, Foekens JA, van Putten WL, van Staveren IL, Portengen H, de Koning WC \& Klijn JG 1992 Prognostic factors in human primary breast cancer: comparison of c-myc and HER2/neu amplification. Journal of Steroid Biochemistry and Molecular Biology 43 13-19. (doi:10.1016/0960-0760(92)90182-I)

Bieker JJ 2001 Krüppel-like factors: three fingers in many pies. Journal of Biological Chemistry 276 34355-34358. (doi:10.1074/jbc.R100043200)

Black AR, Black JD \& Azizkhan-Clifford J 2001 Sp1 and Krüppel-like factor family of transcription factors in cell growth regulation and cancer. Journal of Cellular Physiology 188 143-160. (doi:10.1002/jcp.1111)
Chen C, Bhalala HV, Qiao H \& Dong JT 2002 A possible tumor suppressor role of the KLF5 transcription factor in human breast cancer. Oncogene 21 6567-6572. (doi:10.1038/sj.onc.1205817)

Chen C, Bhalala HV, Vessella RL \& Dong JT 2003 KLF5 is frequently deleted and down-regulated but rarely mutated in prostate cancer. Prostate 55 81-88. (doi:10.1002/ pros.10205)

Chen C, Sun X, Guo P, Dong XY, Sethi P, Cheng X, Zhou J, Ling J, Simons JW, Lingrel JB et al. 2005 Human Krüppel-like factor 5 is a target of the E3 ubiquitin ligase WWP1 for proteolysis in epithelial cells. Journal of Biological Chemistry 280 41553-41561. (doi:10.1074/ jbc.M506183200)

Chen CJ, Lin SE, Lin YM, Lin SH, Chen DR \& Chen CL 2011 Association of expression of Krüppel-like factor 4 and Krüppel-like factor 5 with the clinical manifestations of breast cancer. Pathology Oncology Research 18 161-168. (doi:10.1007/s12253-011-9422-7)

Conkright MD, Wani MA, Anderson KP \& Lingrel JB 1999 A gene encoding an intestinal-enriched member of the Krüppel-like factor family expressed in intestinal epithelial cells. Nucleic Acids Research 27 1263-1270. (doi:10.1093/nar/27.5.1263)

Dong JT \& Chen C 2009 Essential role of KLF5 transcription factor in cell proliferation and differentiation and its implications for human diseases. Cellular and Molecular Life Sciences 66 2691-2706. (doi:10.1007/s00018-009-0045-z)

Ferreira CG, van der Valk P, Span SW, Ludwig I, Smit EF, Kruyt FA, Pinedo HM, van Tinteren H \& Giaccone G 2001 Expression of X-linked inhibitor of apoptosis as a novel prognostic marker in radically resected non-small cell lung cancer patients. Clinical Cancer Research 7 2468-2474.

Frigo DE, Sherk AB, Wittmann BM, Norris JD, Wang Q, Joseph JD, Toner AP, Brown M \& McDonnell DP 2009 Induction of Krüppel-like factor 5 expression by androgens results in increased CXCR4-dependent migration of prostate cancer cells in vitro. Molecular Endocrinology 23 1385-1396. (doi:10.1210/me.2009-0010)

Gregory CW, Whang YE, McCall W, Fei X, Liu Y, Ponguta LA, French FS, Wilson EM \& Earp HS III 2005 Heregulin-induced activation of HER2 and HER3 increases androgen receptor transactivation and CWR-R1 human recurrent prostate cancer cell growth. Clinical Cancer Research 11 1704-1712. (doi:10.1158/10780432.CCR-04-1158)

Guo P, Dong XY, Zhao K, Sun X, Li Q \& Dong JT 2009 Opposing effects of KLF5 on the transcription of MYC in epithelial proliferation in the context of transforming growth factor $\beta$. Journal of Biological Chemistry 284 28243-28252. (doi:10.1074/jbc.M109.036160)

Hammond ME, Hayes DF, Dowsett M, Allred DC, Hagerty KL, Badve S, Fitzgibbons PL, Francis G, Goldstein NS, Hayes M et al. 2010 American Society of Clinical Oncology/College Of American Pathologists guideline recommendations for immunohistochemical 
testing of estrogen and progesterone receptors in breast cancer. Journal of Clinical Oncology 28 2784-2795. (doi:10.1200/JCO.2009.25.6529)

Horwitz KB, Costlow ME \& McGuire WL 1975 MCF-7; a human breast cancer cell line with estrogen, androgen, progesterone, and glucocorticoid receptors. Steroids $\mathbf{2 6}$ 785-795. (doi:10.1016/0039-128X(75)90110-5)

Kenny FS, Hui R, Musgrove EA, Gee JM, Blamey RW, Nicholson RI, Sutherland RL \& Robertson JF 1999 Overexpression of cyclin D1 messenger RNA predicts for poor prognosis in estrogen receptor-positive breast cancer. Clinical Cancer Research 5 2069-2076.

Kwak MK, Lee HJ, Hur K, Park do J, Lee HS, Kim WH, Lee KU, Choe KJ, Guilford P \& Yang HK 2007 Expression of Krüppel-like factor 5 in human gastric carcinomas. Journal of Cancer Research and Clinical Oncology 134 163-167. (doi:10.1007/s00432-007-0265-2)

Liu R, Zheng HQ, Zhou Z, Dong JT \& Chen C 2009 KLF5 promotes breast cell survival partially through fibroblast growth factor-binding protein 1-pERK-mediated dual specificity MKP-1 protein phosphorylation and stabilization. Journal of Biological Chemistry 284 16791-16798. (doi:10.1074/jbc.M808919200)

Liu N, Li H, Li S, Shen M, Xiao N, Chen Y, Wang Y, Wang W, Wang R, Wang Q et al. 2010 The Fbw7/human CDC4 tumor suppressor targets proproliferative factor KLF5 for ubiquitination and degradation through multiple phosphodegron motifs. Journal of Biological Chemistry 285 18858-18867. (doi:10.1074/jbc.M109.099440)

Liu R, Zhou Z, Zhao D \& Chen C 2011 The induction of KLF5 transcription factor by progesterone contributes to progesterone-induced breast cancer cell proliferation and dedifferentiation. Molecular Endocrinology 25 1137-1144. (doi:10.1210/me.2010-0497)

Migliaccio A, Castoria G, Di Domenico M, de Falco A, Bilancio A, Lombardi M, Barone MV, Ametrano D, Zannini MS, Abbondanza C et al. 2000 Steroid-induced androgen receptor-oestradiol receptor $\beta$-Src complex triggers prostate cancer cell proliferation. EMBO Journal 19 5406-5417. (doi:10.1093/emboj/19.20.5406)

Naderi A \& Hughes-Davies L 2008 A functionally significant cross-talk between androgen receptor and ErbB2 pathways in estrogen receptor negative breast cancer. Neoplasia 10 542-548.

Nakajima Y, Akaogi K, Suzuki T, Osakabe A, Yamaguchi C, Sunahara N, Ishida J, Kako K, Ogawa S, Fujimura T et al. 2011 Estrogen regulates tumor growth through a nonclassical pathway that includes the transcription factors ER $\beta$ and KLF5. Science Signaling 4 ra22. (doi:10.1126/scisignal.2001551)

Nandan MO, McConnell BB, Ghaleb AM, Bialkowska AB, Sheng H, Shao J, Babbin BA, Robine S \& Yang VW 2008 Krüppel-like factor 5 mediates cellular transformation during oncogenic KRAS-induced intestinal tumorigenesis. Gastroenterology 134 120-130. (doi:10.1053/ j.gastro.2007.10.023)
Ni M, Chen Y, Lim E, Wimberly H, Bailey ST, Imai Y, Rimm DL, Liu XS \& Brown M 2011 Targeting androgen receptor in estrogen receptor-negative breast cancer. Cancer Cell 20 119-131. (doi:10.1016/j.ccr.2011. 05.026)

Shi H, Zhang Z, Wang X, Liu S \& Teng CT 1999 Isolation and characterization of a gene encoding human Krüppellike factor 5 (IKLF): binding to the CAAT/GT box of the mouse lactoferrin gene promoter. Nucleic Acids Research 27 4807-4815. (doi:10.1093/nar/27.24.4807)

Soon MS, Hsu LS, Chen CJ, Chu PY, Liou JH, Lin SH, Hsu JD \& Yeh KT 2011 Expression of Krüppel-like factor 5 in gastric cancer and its clinical correlation in Taiwan. Virchows Archiv 459 161-166. (doi:10.1007/s00428-011-1111-0)

Sun R, Chen X \& Yang VW 2001 Intestinal-enriched Krüppel-like factor (Krüppel-like factor 5) is a positive regulator of cellular proliferation. Journal of Biological Chemistry 276 6897-6900. (doi:10.1074/jbc. C000870200)

Suzuki T, Miki Y, Takagi K, Hirakawa H, Moriya T, Ohuchi N \& Sasano H 2010 Androgens in human breast carcinoma. Medical Molecular Morphology 43 75-81. (doi:10.1007/ s00795-010-0494-3)

Takagi K, Miki Y, Nagasaki S, Hirakawa H, Onodera Y, Akahira J, Ishida T, Watanabe M, Kimijima I, Hayashi S et al. 2010 Increased intratumoral androgens in human breast carcinoma following aromatase inhibitor exemestane treatment. Endocrine-Related Cancer 17 415-430. (doi:10.1677/ERC-09-0257)

Tong D, Czerwenka K, Heinze G, Ryffel M, Schuster E, Witt A, Leodolter S \& Zeillinger R 2006 Expression of KLF5 is a prognostic factor for disease-free survival and overall survival in patients with breast cancer. Clinical Cancer Research 12 2442-2448. (doi:10.1158/1078-0432.CCR-05-0964)

Yang Y, Goldstein BG, Chao HH \& Katz JP 2005 KLF4 and KLF5 regulate proliferation, apoptosis and invasion in esophageal cancer cells. Cancer Biology \& Therapy 4 1216-1221. (doi:10.4161/cbt.4.11.2090)

Zhao D, Zheng HQ, Zhou Z \& Chen C 2010 The Fbw7 tumor suppressor targets KLF5 for ubiquitin-mediated degradation and suppresses breast cell proliferation. Cancer Research 70 4728-4738. (doi:10.1158/00085472.CAN-10-0040)

Zheng HQ, Zhou Z, Huang J, Chaudhury L, Dong JT \& Chen C 2009 Krüppel-like factor 5 promotes breast cell proliferation partially through upregulating the transcription of fibroblast growth factor binding protein 1. Oncogene 28 3702-3713. (doi:10.1038/onc.2009.235)

Received in final form 3 August 2012

Accepted 30 August 2012

Made available online as an Accepted Preprint 30 August 2012 